\title{
Review of: "Ectopic endometriosis, menstruation, and acute appendicitis: A thought-provoking case"
}

\section{francesco coratti}

Potential competing interests: The author(s) declared that no potential competing interests exist.

Well structured article but of little scientific interest. However it demonstrates what can be found in the course of appendicitis 